Z Rheumatol 2016 $\cdot 75: 63-68$

DOI 10.1007/s00393-016-0044-z

Online publiziert: 26. Januar 2016

๑) Springer-Verlag Berlin Heidelberg 2016

\section{Redaktion}

H.-P. Tony, Würzburg

CrossMark

\author{
M. Schmalzing ${ }^{1} \cdot$ M. Krockenberger ${ }^{2} \cdot$ A. Honig ${ }^{3} \cdot$ H.-P. Tony ${ }^{1}$ \\ ${ }^{1}$ Rheumatologie/klinische Immunologie, Medizinische Klinik und Poliklinik 2, Zentrum Innere Medizin, \\ Universitätsklinikum Würzburg, Würzburg, Deutschland \\ ${ }^{2}$ Frauenklinik und Poliklinik, Universitätsklinikum Würzburg, Würzburg, Deutschland \\ ${ }^{3}$ Klinik für Frauenheilkunde und Geburtshilfe, Katholisches Klinikum Mainz, Mainz, Deutschland
}

\title{
Gynäkologische Malignome bei Patientinnen mit rheumatisch-entzündlichen Systemerkrankungen
}

Zervixkarzinome und andere Genitalkarzinome treten bei bestimmten $\mathrm{Au}$ toimmunerkrankungen, v. a. beim systemischen Lupus erythematodes (SLE), etwas häufiger auf. Dagegen ist die Inzidenz für andere gynäkologische Tumoren wie Mammakarzinom, Ovarialkarzinom und das Endometriumkarzinom bei Patientinnen mit Autoimmunerkrankungen eher geringer $[1,2]$.

Allerdings wurde für Patientinnen mit rheumatoider Arthritis gezeigt, dass die Prognose eines Mammakarzinoms deutlich schlechter ist und mit erhöhter Mortalität einhergeht [3]. Daraus ergibt sich, dass eine effektive Screeningstrategie für diese Patientinnen besonders wichtig ist.

\section{》) Das Immunsystem spielt bei der Tumorkontrolle eine bedeutende Rolle}

Für die meisten gynäkologischen Tumoren existieren Hinweise, dass das Immunsystem bei der Tumorkontrolle eine bedeutende Rolle spielt. Welchen Einfluss immunsuppressive Medikamente oder Biologika-DMARDs („disease modifying antirheumatic drugs“) in diesem Prozess haben, ist schwer zu analysieren und nicht abschließend geklärt.

Aufgrund ihrer Häufigkeit werden in diesem Artikel das Mammakarzinom, das Zervixkarzinom und das Ovarialkarzinom behandelt. Das Endometri- umkarzinom sowie das Vulva- und das Vaginalkarzinom finden keine Erwähnung.

\section{Hintergrund}

\section{Mammakarzinom}

In den letzten Jahren hat sich herauskristallisiert, dass auch beim Mammakarzinom - dem häufigsten Malignom der Frau - eine Immunabhängigkeit nachzuweisen ist, die für neue therapeutische Optionen genutzt werden kann. So gibt es beim metastasierten triple-negativen Mammakarzinom erste vielversprechende Ergebnisse zur Therapie der Erkrankung mit Immuncheckpoint-Inhibitoren wie Pembrolizumab (PD1-Blockade) [4].

Kato et al. [5] haben am Beispiel der Nierentransplantation, die in der Regel im Vergleich zu Autoimmunerkrankungen eine intensivere Immunsuppression beinhaltet, die Situation im Hinblick auf das Mammakarzinom in einer aktuellen Arbeit beleuchtet. Mammakarzinome treten bei diesen Patientinnen nicht nur häufiger auf, sondern zeichnen sich auch oft durch ein aggressiveres Verhalten aus.

\section{Zervixkarzinom}

Das Zervixkarzinom gilt als der dritthäufigste genitale Tumor einer Frau in Deutschland. Glücklicherweise sinken bei diesem Tumor die Inzidenzen auf- grund einer effektiven Vorsorge und der Möglichkeit zur Vakzinierung gegen auslösende humane Papillomaviren (HPV). Die Impfung soll dabei die Entwicklung ausgehend von einer Infektion mit HPV über eine Vorstufe bis hin zum invasiven Karzinom verhindern.

Es ist zu befürchten, dass eine medikamentöse Immunsuppression - sicherlich in Abhängigkeit von der Intensität und der Präparatewahl - diesen Prozess ungünstig beeinflussen kann [6, 7]. So zeigt sich auch am Beispiel der Nierentransplantation ein stark erhöhtes Risiko für HPV-assoziierte Karzinome (14-fach für Zervixkarzinome, 50-fach für Vulvakarzinome und 100-fach für Analkarzinom) [8]. Das Zervixkarzinom stellt somit das gynäkologische Malignom mit dem am besten belegten Einfluss von Immunsuppression in der Karzinogenese dar.

Aufgrund der vorliegenden Beobachtungen scheint ein langjähriger Einsatz von Immunsuppressiva zumindest bei Patientinnen mit SLE das Risiko für zervikale Präkanzerosen zu erhöhen [9, 10]. Allerdings fand sich in einer aktuellen Analyse des dänischen Biologikaregisters und in 2 kleineren Studien unter Biologika kein erhöhtes Risiko einer Progression einer zervikalen Dysplasie oder eines zervikalen Carcinoma in situ in ein Zervixkarzinom [11-13]. 
Tab. 1 Nachsorge nach Zervixkarzinom gemäß S3-Leitlinie: Obligate lokoregionäre Nachsorgeuntersuchungen und Intervalle

\begin{tabular}{|lll|}
\hline Untersuchung & 1. bis 3. Jahr & 4. und 5. Jahr \\
\hline Anamnese & Kontrollen 3-monatlich & Kontrollen 6-monatlich \\
\hline Klinische Untersuchung & Kontrollen 3-monatlich & Kontrollen 6-monatlich \\
\hline $\begin{array}{l}\text { Spekulumeinstellung und } \\
\text { Zytologie (Pap) }\end{array}$ & Kontrollen 3-monatlich & Kontrollen 6-monatlich \\
\hline
\end{tabular}

Tab. 2 Nachsorge nach Ovarialkarzinom gemäß S3-Leitlinie

\begin{tabular}{llll}
\hline Untersuchung & Jahr 1-3 & Jahr 4-5 & Jahr 5-10 \\
\hline $\begin{array}{l}\text { Rektovaginale Palpati- } \\
\text { on, Kolposkopie }\end{array}$ & Alle 3 bis 4 Monate & Alle 6 Monate & Jährlich \\
\hline Zytologie der Vagina & Nein & Nein & Nein \\
\hline Labor Ca 125 & Alle 3 bis 4 Monate & Alle 6 Monate & Jährlich \\
\hline Vaginale Sonographie & Alle 3 bis 4 Monate & Alle 6 Monate & Jährlich \\
\hline
\end{tabular}

'Wenn präoperativ erhöht; kann in Absprache mit Patientin auch weggelassen werden, da kein

Benefit bezüglich Überleben belegt ist.

\section{Ovarialkarzinom}

Das Ovarialkarzinom stellt das zweithäufigste gynäkologische Malignom in unseren Breiten dar. Für seine Entstehung sind unter anderem genetische Dispositionen bekannt, deren Vorhandensein eine Hochrisikopopulation definiert. Da die Erkrankung oft erst in einem späteren Stadium diagnostiziert wird, ist meist von einer schlechten Prognose auszugehen. Bezüglich der Tumorentstehung gibt es einerseits Hinweise, dass das Immunsystem diese verhindert, andererseits kann ein entzündliches Tumormikromilieu auch zur Karzinogenese beitragen [14].

\section{Tumorvorsorge}

\section{Mammakarzinom}

Die klinische Untersuchung der Brust durch einen Arzt ist Bestandteil des gesetzlichen Krebsfrüherkennungsprogramms ab dem 30. Lebensjahr.

Brustkrebs tritt am häufigsten zwischen der 6. und 7. Lebensdekade auf. Seit dem Jahr 2009 ist der Aufbau des nationalen Mammographie-Screeningprogramms mit wohnortnaher Versorgung abgeschlossen. Frauen zwischen dem 50. und 70. Lebensjahr werden alle 2 Jahre zur Mammographie durch die jeweiligen Screeningeinheiten eingeladen. Das Screeningalter ist dem zweigipfligen Inzidenzmaximum des Mammakarzinoms geschuldet. Das nationale Mammographie-Screeningprogramm umfasst aber lediglich die Röntgenaufnahme der weiblichen Brust [15].

\section{Modifikation der Vorsorgeemp- fehlungen bei immunsuppressiver oder immunmodulatorischer Therapie}

Es gibt zum jetzigen Zeitpunkt keine speziellen Vorsorgeempfehlungen bei Patientinnen unter immunsuppressiver oder immunmodulatorischer Therapie, obschon diese vor dem Hintergrund der rezent erhobenen Daten zum Zusammenhang zwischen Tumor und Immunsystem möglicherweise als sinnvoll anzusehen wären.

\section{Zervixkarzinom}

Für ca. $75 \%$ der zervikalen intraepithelialen Neoplasien (CIN) als Vorstufe des Zervixkarzinoms sind die Highrisk-HPV-Typen 16 und 18 verantwortlich. Die HPV-Infektion kann durch Impfung verhindert werden. In den Impfempfehlungen der Ständigen Impfkommission (STIKO) vom August 2014 ist je nach verwendetem Impfstoff eine HPV-Impfung für Mädchen im Alter von 9 bis 14 (Cervarix $^{\circledR}$ ) bzw. 9 bis 13 Jahren $\left(\right.$ Gardasil $\left.^{\circledR}\right)$ vorgesehen. Es handelt sich um 2 Dosen im Abstand von 6 Monaten. Eine Nachholimpfung bei nicht geimpften oder unvollständig geimpften Mädchen kann gemäß dieser Empfehlung bis zur Vollendung des 18. Lebensjahres (also bis zum Tag vor dem 18. Geburtstag) stattfinden. Die vollständige Impfserie sollte vor dem ersten Geschlechtsverkehr abgeschlossen sein. Für Frauen, die älter als 17 Jahre sind und keine Impfung gegen HPV erhalten haben, weist die STIKO darauf hin, dass sie ebenfalls von einer Impfung gegen HPV profitieren können. Es liege in der Verantwortung des Arztes, nach individueller Prüfung von Nutzen und Risiko der Impfung seine Patientinnen auf der Basis der Impfstoffzulassung darauf hinzuweisen.

Es konnte gezeigt werden, dass die Impfung gegen HPV das Auftreten von zervikalen Präkanzerosen und von Zervixkarzinom reduziert. Es existieren sogar Hinweise, dass sich die Impfung bei bereits nachgewiesener HPV-Infektion günstig auf das Karzinomrisiko auswirken kann [17, 18].

In Deutschland soll ein jährliches Screening für Zervixkarzinom mittels Pap-Abstrich definitiv ab dem 20. Lebensjahr oder 3 Jahre nach Aufnahme des vaginalen Geschlechtsverkehrs beginnen [19]. Diese Empfehlungen gelten im Falle eines unauffälligen Pap-Abstriches dauerhaft. Bei Auffälligkeiten des Pap-Abstrichs kann den Empfehlungen gemäß der Münchner Nomenklatur III zur Einteilung der Pap-Abstriche gefolgt werden [20]. Je nach Pap-Befund wird zu zytologischen Kontrollen nach bestimmten Intervallen, zu Differenzialkolposkopien oder zur histologischen Sicherung geraten.

\section{Modifikation der Vorsorgeemp- fehlungen bei immunsuppressiver oder immunmodulatorischer Therapie}

Eine kürzlich erschienene Übersichtsarbeit nimmt zum ZervixkarzinomScreening bei Immunsupprimierten Stellung und weist aber darauf hin, dass nur für HIV-Infizierte diesbezüglich eine suffiziente Datenlage existiert [21]. Diese Patientengruppe sollte nach Ansicht der Autoren nach Erstdiagnose alle 6 Monate im ersten Jahr gescreent werden und danach jährlich. In Analogie dazu wird ein Screening bei SLE-Patien- 
tinnen diskutiert, da für diese Population ebenfalls erhöhte Zervixkarzinomraten bekannt sind

Bezüglich der HPV-Impfung verweist die European League Against Rheumatism (EULAR) auf die besondere Bedeutung der Impfung bei SLE-Patientinnen und diskutiert eine Impfung bei Erwachsenen bis zum Alter von 25 Jahren. Die Impfung in dieser Altersgruppe ist unabhängig vom SLE in Studien gut belegt [22].

\section{Ovarialkarzinom}

Studien zum intensivierten Screening haben sich auch bei Risikopersonen nicht als effektiv erwiesen. Daher gibt es derzeit keine Empfehlung zur Vorsorge beim Ovarialkarzinom - weder in der Normalbevölkerung noch bei immunsupprimierten Patientinnen oder bei Hochrisikopersonen. Sollte eine familiäre Hochrisikosituation auf Basis einer bestimmten Mutation (BRCA1/2) nachgewiesen sein, kann nach abgeschlossener Familienplanung allerdings eine prophylaktische Adnexektomie beidseits unter Beachtung der veränderten Lebensqualität diskutiert werden [23].

\section{Tumornachsorge}

Zur Tumornachsorge bei gynäkologischen Malignomen existieren bislang keine validen Daten, die ein Abweichen vom üblichen Vorgehen bei Patientinnen mit rheumatisch entzündlichen Erkrankungen rechtfertigen würden. Allerdings sollte gerade dieses Patientenkollektiv zu einer besonders konsequenten Nachsorge motiviert werden, weshalb die etablierten Empfehlungen im Folgenden dargestellt werden.

\section{Mammakarzinom}

Die Nachsorge bei Mammakarzinom beginnt mit der abgeschlossenen lokoregionären Primärbehandlung. Sie besteht aus Anamnese, körperlicher Untersuchung, ärztlicher Beratung und Begleitung sowie bildgebender Diagnostik zur Erkennung eines lokoregionären Rezidivs. Bei auffälligem Befund wird die Nachsorge symptomorientiert konzipiert [15, 24, 25].

Z Rheumatol 2016 · 75:63-68 DOI 10.1007/s00393-016-0044-Z

(c) Springer-Verlag Berlin Heidelberg 2016

M. Schmalzing · M. Krockenberger · A. Honig · H.-P. Tony

Gynäkologische Malignome bei Patientinnen mit
rheumatisch-entzündlichen Systemerkrankungen

\section{Zusammenfassung}

Das Risiko für gynäkologische Tumoren bei rheumatisch-entzündlichen Systemerkrankungen scheint nur in Bezug auf das Zervixkarzinom v. a. bei systemischem Lupus erythematodes erhöht zu sein. Studien weisen auf die Bedeutung des Immunsystems für die Tumorkontrolle bei gynäkologischen Tumoren hin. Bislang konnte aber nur für das Zervixkarzinom ein ungünstiger Einfluss einer medikamentösen Immunsuppression bei rheumatologischen Patientinnen gezeigt werden. Biologika wirken sich bei dieser Entität dagegen nicht eindeutig tumorfördernd aus. Die Datenlage bei dieser Fragestellung ist allerdings begrenzt. Generelle Screeningempfehlungen existieren für das Mammakarzinom und das Zervixkarzinom. Empfehlungen zur Tumornachsorge werden dargestellt. Aufgrund der unzureichenden Datenlage empfiehlt sich zumindest in den ersten 5 Jahren nach kurativer onkologischer Therapie eine sehr strenge Indikationsstellung zum Einsatz von Immunsuppressiva und Biologika auch bei gynäkologischen Tumoren. Im Falle des Mammakarzinoms wird sogar die Beachtung eines längeren Intervalls diskutiert.

\section{Schlüsselwörter}

Immunsuppression · Mammakarzinom · Ovarialkarzinom · Zervixkarzinom · Systemischer Lupus erythematodes

\section{Gynecological cancers in patients with inflammatory rheumatic diseases}

\section{Abstract}

The risk of gynecological cancers in patients with inflammatory rheumatic diseases only seems to be elevated with respect to cervical cancer and mainly in patients with systemic lupus erythematosus. There is increasing evidence for an influence of the immune system on tumor control of gynecological malignancies; however, an adverse influence of immunosuppressive treatment in rheumatic patients was indicated only for the risk of cervical cancer. In contrast, biologics could not be shown to cause an increased risk of cervical cancer but data on this topic are limited. General screening recommendations exist for breast cancer and cervical cancer. Recommendations for follow-up after oncological treatment are presented. Because of limited evidence immunosuppressive and biological treatment should be applied with great restraint at least within the first 5 years after curative oncological treatment also for gynecological tumors. As far as breast cancer is concerned an even longer interval is under discussion.

\section{Keywords}

Immunosuppression · Breast cancer · Ovarian cancer · Cervical cancer · Systemic lupus erythematosus
Bei symptomfreien Frauen nach abgeschlossener brusterhaltender Therapie ist zur Rezidivdiagnostik die regelmäßig vorzunehmende apparative Diagnostik (Mammographie, Sonographie) im Bereich der ipsilateralen Brust angezeigt [27, 28].

Die Tumornachsorge beim Mammakarzinom ist generell engmaschig und langfristig. In mindestens $75 \%$ der Fälle erstreckt sich die durchgeführte antihormonelle Therapie über 5 bis 10 Jahre, sodass sich die Patientin diesbezüglich relativ lange in regelmäßiger fachärztlicher Kontrolle befindet.

\section{Zervixkarzinom}

Die geltende S3-Leitlinie zur Diagnostik, Therapie und Nachsorge des Zervixkarzinoms definiert unabhängig von einer bestehenden Immunsuppression Nachsorgeintervalle nach erfolgter Therapie ([29], - Tab. 1). Es ist anzunehmen, dass bei Patientinnen mit rheumatischen Erkrankungen eine schlechtere Prognose 
der Tumorerkrankung als im Vergleichskollektiv besteht [30].

\section{Ovarialkarzinom}

Zur Tumornachsorge bei Patientinnen mit Zustand nach Ovarialkarzinom existieren ebenfalls Empfehlungen im Rahmen der S3-Leitlinie [23]. Die routinemäßige Tumormarkerbestimmung oder der routinemäßige Einsatz von apparativer Diagnostik erbringt hierbei allerdings keinen Vorteil bezüglich der Gesamtprognose der Erkrankung. Empfohlen sind eine regelmäßige sorgfältige Anamnese, die körperliche Untersuchung inklusive gynäkologischer Spiegel- und Tastuntersuchung, die rektale Untersuchung und die Vaginalsonographie.

Bezüglich der Frequenz der Nachsorgeuntersuchungen existieren keine einheitlichen Empfehlungen deutscher Fachgesellschaften. Daher bietet die unten stehende Tabelle der Schweizer Kollegen eine Orientierungshilfe für die Nachsorge beim Ovarialkarzinom (Empfehlungen gynäkologisch onkologische Nachsorge der AGO (Arbeitsgemeinschaft für Gynäkologische Onkologie und Brustgesundheit) der Schweizerischen Gesellschaft für Gynäkologie und Geburtshilfe, Stand März 2009, • Tab. 2).

\section{Daten und Empfehlungen zur Immunsuppression oder DMARD-Therapie bei Malignomanamnese}

Vor dem Hintergrund der Beobachtung, dass gerade für das Mammakarzinom die Rezidivrate nach Nierentransplantation mit $23 \%$ besonders hoch erscheint, ist zumindest in den ersten 5 Jahren nach Karzinomdiagnose erhöhte Vorsicht bei immunsuppressiver Therapie geboten und eine strengere Indikationsstellung zu fordern [31]. Allerdings weisen immunsuppressive oder DMARD-Therapien in der Rheumatologie eine viel geringere Intensität auf als Therapieregime in der Transplantationsmedizin.

Raaschou et al. [32] haben Patientinnen mit rheumatoider Arthritis und vordiagnostiziertem Brustkrebs in den nationalen schwedischen Registern analysiert. Diejenigen Patientinnen, die eine
TNF-Inhibitor-Therapie erhielten, zeigten keine höhere Tumorrezidivrate als $\mathrm{Pa}$ tientinnen, die konventionelle Basistherapeutika einnahmen. Allerdings betrug der mediane zeitliche Abstand zwischen Mammakarzinomdiagnose und dem Beginn der TNF-Inhibitortherapie 9,4 Jahre, und es ist anzunehmen, dass Patientinnen, die im Register dokumentiert wurden, vor TNF-inhibitorischer Therapie besonders sorgfältig in Bezug auf ein Mammakarzinomrezidivrisiko ausgewählt wurden.

\section{》) In den ersten 5 Jahren nach Karzinomdiagnose ist erhöhte Vorsicht geboten}

Im britischen Register zeigte sich bei Patientinnen ohne Malignomanamnese sogar ein Trend $\mathrm{zu}$ einem niedrigeren De-novo-Mammakarzinomrisiko unter TNF-Inhibitoren. In einer großen aktuellen Metaanalyse war das Risiko für inzidente Mammakarzinome ebenfalls nicht erhöht [33, 34].

Dennoch wird in der Literatur häufig bei stattgehabtem Mammakarzinom sogar ein Intervall von 10 Jahren zwischen Tumortherapie und immunsuppressiver bzw. Biologika-DMARD-Therapie diskutiert. Diese Empfehlung gründet lediglich auf die bekannte Neigung zu späten Rezidiven und nicht auf Studien, die Hinweise auf ein erhöhtes Rezidivrisiko bei kürzerem Intervall gezeigt hätten.

Ansonsten sei an dieser Stelle auf den Übersichtsvortrag von Schmalzing et al. in diesem Themenheft verwiesen.

\section{Fazit für die Praxis}

- Studien weisen auf die Bedeutung des Immunsystems für die Tumorkontrolle bei gynäkologischen Tumoren hin. Bislang konnte aber nur für das Zervixkarzinom ein ungünstiger Einfluss einer medikamentösen Immunsuppression gezeigt werden. Biologika wirken sich bei dieser Entität dagegen nicht eindeutig tumorfördernd aus.

- Das Risiko für gynäkologische Tumoren bei entzündlich rheumatischen
Systemerkrankungen scheint nur in Bezug auf das Zervixkarzinom v. a. bei SLE erhöht zu sein.

- Generelle Screeningempfehlungen existieren für das Mammakarzinom und das Zervixkarzinom. Für das Zervixkarzinom muss ein besonders engmaschiges Screening unter Immunsuppressiva diskutiert werden.

- Aufgrund der unzureichenden Datenlage empfiehlt sich zumindest in den ersten 5 Jahren nach kurativer Therapie eine sehr strenge Indikationsstellung zum Einsatz von Immunsuppressiva und Biologika bei gynäkologischen Tumoren.

Korrespondenzadresse
Rheumatologie/klinische
Immunologie, Medizinische
Klinik und Poliklinik 2,
Zentrum Innere Medizin,
Universitätsklinikum
Würzburg
Oberdürrbacher Str. 6,
97080 Würzburg,
Deutschland
schmalzing_m@ukw.de

\section{Einhaltung ethischer Richtlinien}

Interessenkonflikt. Dieses Manuskript entstand auf Basis einer von Roche und Chugai unterstützten Arbeitsgruppe (Systemerkrankung RA), aber ohne jegliche Einflussnahme der Firmen auf das Ergebnis oder die Publikation. M. Schmalzing hat Vortrags-und/ oder Beratungshonorare erhalten von AbbVie, Actelion, BMS, Chugai, Genzyme, Janssen-Cilag, Novartis, Pfizer, Roche, UCB. M. Krockenberger hat Vortragsund/oder Beratungshonorare erhalten von Chugai. A. Honig hat Vortrags- und/oder Beratungshonorare erhalten von Amgen, Celgene, Chugai, MSD, Pfizer, Roche, Astra Zeneca, Novartis. H.-P. Tony hat Vortragsund/oder Beratungshonorare erhalten von AbbVie, Chugai, Janssen-Cilag, MSD, Pfizer, Roche, UCB.

Dieser Beitrag beinhaltet keine Studien an Menschen oder Tieren.

\section{Literatur}

1. Hemminki K, Liu X, Ji J, Försti A, Sundquist J, Sundquist K (2012) Effect of autoimmune diseases on risk and survival in female cancers. Gynecol Oncol 127(1):180-185

2. Smitten AL, Simon TA, Hochberg MC, Suissa $S$ (2008) A meta-analysis of the incidence of malignancy in adult patients with rheumatoid arthritis. Arthritis Res Ther 10(2):R45 
3. Ji J, Liu X, Sundquist K, Sundquist J (2011) Survival of cancer in patients with rheumatoid arthritis: a follow-up study in Sweden of patients hospitalized with rheumatoid arthritis 1 year before diagnosis of cancer. Rheumatology (Oxford) 50(8):1513-1518

4. Emens LA (2012) Breast cancer immunobiology driving immunotherapy: vaccines and immune checkpoint blockade. Expert Rev Anticancer Ther 12(12):1597-1611

5. Kato T, Kakuta Y, Yamanaka K, Okumi M, Abe T, Imamura R, Ichimaru N, Takahara S, Nonomura $\mathrm{N}$ (2015) Early diagnosis and treatment of breast cancer in Japanese kidney transplant recipients: a single center experience. Springerplus 4:196

6. Likes W, Santoso JT, Wan J (2013) A cross-sectional analysis of lower genital tract intraepithelial neoplasia in immune-compromised women with an abnormal pap. Arch Gynecol Obstet 287(4):743-747

7. Kobayashi A, Weinberg V, Darragh T, SmithMcCune K (2008) Evolving immunosuppressive microenvironment during human cervical carcinogenesis. Mucosal Immunol 1(5):412-420

8. Hinten $F$, Meeuwis KA, van Rossum MM, de Hullu JA (2012) HPV-related (pre)malignancies of the female anogenital tract in renal transplant recipients. Crit Rev Oncol Hematol 84(2):161-180

9. Klumb EM, Araújo ML Jr, Jesus GR, Santos $D B$, Oliveira AV, Albuquerque EM, Macedo JM (2010) Is higher prevalence of cervical intraepithelial neoplasia in women with lupus due to immunosuppression? J Clin Rheumatol 16(4):153-157

10. Dugué PA, Rebolj M, Garred P, Lynge E (2013) Immunosuppression and risk of cervical cancer Expert Rev Anticancer Ther 13(1):29-42

11. Cordtz R, Mellemkjær L, Glintborg B, Hetland ML, Dreyer L (2015) Malignant progression of precancerous lesions of the uterine cervix following biological DMARD therapy in patients with arthritis. Ann Rheum Dis 74(7):1479-1480

12. Mercer LK, Low AS, Galloway JB et al (2013) AntiTNF therapy in women with rheumatoid arthritis with a history of carcinoma in situ of the cervix. Ann Rheum Dis 72:143-144

13. Kim SC, Schneeweiss S, Liu J et al (2014) Biologic disease-modifying antirheumatic drugs and risk of high-grade cervical dysplasia and cervical cancer in women with rheumatoid arthritis. Arthritis Rheumatol66(Suppl):P895

14. Krockenberger $M$, Dombrowski $Y$, Weidler $C$, Ossadnik $\mathrm{M}$, Honig A, Häusler $\mathrm{S}$, Voigt $\mathrm{H}$, Becker JC, Leng L, Steinle A, Weller M, Bucala R, Dietl J, Wischhusen JJ (2008) Macrophage migration inhibitory factor contributes to the immune escape of ovarian cancer by down-regulating NKG2D. Immunol 180(11):7338-7348

15. S3-Leitlinie Mammakarzinom der Frau. Diagnostik Therapie und Nachsorge. AWMF-Registernummer: 032-045OL

16. Aktualisierte Empfehlungen der Kommission Uterus auf Grundlage der S2k Leitlinie Herausgegeben von der Kommission Uterus der Arbeitsgemeinschaft Gynäkologische Onkologie e.V.

17. Empfehlungen der Ständigen Impfkommission (STIKO) am Robert Koch-Institut (2014) Epidemiologisches Bulletin 25. August 2014/34. Stand: August 2014

18. Kash N, Lee MA, Kollipara R, Downing C, Guidry J, Tyring SK (2015) Safety and efficacy data on vaccines and immunization to human Papillomavirus. JClin Med 4(4):614-633
19. Prävention, Diagnostik und Therapie der HPVInfektion und präinvasiver Läsionen des weiblichen Genitale. AWMF- Leitlinienregisternummer 015/027

20. Griesser et al (2014) Münchner Nomenklatur III für die gynäkologische Zytodiagnostik der Zervix ab 1. Juli2014. Frauenarzt 54:1042-1048

21. Nguyen ML, Flowers L (2014) Cervical cancer screening in immunocompromised women. Obstet Gynecol Clin North Am 40(2):339-357

22. van Assen S, Agmon-Levin N, Elkayam O, Cervera R, Doran MF, Dougados M, Emery P, Geborek P, loannidis JP, Jayne DR, Kallenberg CG, Müller-Ladner U, Shoenfeld Y, Stojanovich L, Valesini G, Wulffraat NM, Bijl M (2011) EULAR recommendations for vaccination in adult patients with autoimmune inflammatory rheumatic diseases. Ann Rheum Dis 70(3):414-422

23. S3-Leitlinie Diagnostik, Therapie und Nachsorge maligner Ovarialtumoren (2013) Version 1.0/Juni 2013. AWMF-Leitlinienregisternummer 032/0350L

24. Rojas MP, Telaro E, Russo A, Moschetti I, Coe L, Fossati R, Palli D, del Roselli TM, Liberati A (2005) Follow-up strategies for women treated for early breast cancer. Cochrane Database Syst Rev Jan 25(1):CD001768

25. Grunfeld E, Dhesy-Thind S, Levine M (2005) Clinical practice guidelines for the care and treatment of breast cancer: follow-up after treatment for breast cancer (summary of the 2005 update). CMAJ 172(10):1319-1320

26. Selby P, Gillis C, Haward R (1996) Benefits from specialised cancer care. Lancet 348(9023):313-318

27. Grunfeld E, Noorani H, McGahan L, Paszat L, Coyle D, van Walraven C, Joyce J, Sawka C (2002) Surveillance mammography after treatment of primary breast cancer: a systematic review. Breast 11(3):228-235

28. Khatcheressian JL, Hurley P, Bantug E, Esserman LJ, Grunfeld E, Halberg F, Hantel A, Henry NL, Muss HB, Smith TJ, Vogel VG, Wolff AC, Somerfield MR, Davidson NE (2013) Breast cancer follow-up and management after primary treatment: American Society of Clinical Oncology clinical practice guideline update. JClin Oncol 31(7):961-965

29. Diagnostik, Therapie und Nachsorge der Patientin mit Zervixkarzinom. AWMF-Leitlinienregisternummer:032-0330L

30. Ji J, Liu X, Sundquist K, Sundquist J (2011) Survival of cancer in patients with rheumatoid arthritis: a follow-up study in Sweden of patients hospitalized with rheumatoidarthritis 1 yearbefore diagnosis of cancer. Rheumatology (Oxford) 50(8):1513-1518

31. Penn I (1997) Evaluation of transplant candidates with pre-existing malignancies. Ann Transplant 2(4):14-17

32. Raaschou P, Frisell T, Askling J (2015) TNF inhibitor therapy and risk of breast cancer recurrence in patients with rheumatoid arthritis: a nationwide cohort study. Ann Rheum Dis 74(12):2137-2143

33. Mercer LK, Lunt M, Low AL, Dixon WG, Watson KD, Symmons DP, Hyrich KL (2015) Risk of solid cancer in patients exposed to anti-tumour necrosis factor therapy: results from the british society for rheumatology biologics register for rheumatoid arthritis. Ann Rheum Dis 74(6):1087-1093

34. Liu Y, Fan W, Chen H, Yu MX (2014) Risk of breast cancer and total malignancies in rheumatoid arthritis patients undergoing TNF-a antagonist therapy: a meta-analysis of randomized control trials. Asian Pac J Cancer Prev 15(8):3403-3410

\section{Zweitmeinung vor Klinik-Op gefragt}

Vor einer geplanten Operation in einem Krankenhaus ziehen drei von vier Patienten in Betracht, sich eine ärztliche Zweitmeinung einzuholen. Das ist das Ergebnis einer aktuellen Forsa-Umfrage im Auftrag des Wissenschaftlichen Instituts für Qualität und Effizienz im Gesundheitswesen (WINEG) der Techniker Krankenkasse (TK). Danach gaben 74 Prozent der 1000 befragten gesetzlich Versicherten an, eine Zweitmeinung zu erfragen, würde ihr Arzt eine Operation oder Untersuchung in einer Klinik vorschlagen. Groß ist auch das Interesse an möglichen negativen Auswirkungen von „zu viel Behandlung". 85 Prozent der Befragten bezeichneten es laut Mitteilung als hilfreich, mehr über diesen Aspekt zu erfahren. Damit einher geht die Vermutung, möglicherweise schon einmal mehr als die wirklich notwendige Diagnostik und Therapie erhalten zu haben. Jeder vierte Befragte (27 Prozent) hat manchmal oder sogar häufig den Eindruck, dass ein Arzt eine unnötige Behandlung oder Untersuchung empfiehlt, wobei ein Drittel dieser Betroffenen angibt, sich trotz Bedenken behandeln lassen zu haben. Die Umfrageergebnisse zeigen, dass in Sachen Überversorgung ein Problembewusstsein besteht, resümiert die TK. Das GKV-Versorgungsstärkungsgesetz sieht vor, dass Patienten mindestens zehn Tage vor einer Operation vom Arzt über ihr Recht auf eine unabhängige Zweitmeinung aufgeklärt werden müssen.

Quelle:www.tk.de 
Hier steht eine Anzeige.

算 Springer 